\title{
Genetic risk for myocardial infarction in Japanese individuals with or without chronic kidney disease
}

\author{
TETSUO FUJIMAKI ${ }^{1}$, KIMIHIKO KATO ${ }^{1}$, KIYOSHI YOKOI $^{1}$, TETSURO YOSHIDA ${ }^{2}$, MITSUTOSHI OGURI $^{3}$, \\ SACHIRO WATANABE ${ }^{4}$, NORIFUMI METOKI ${ }^{5}$, HIDEMI YOSHIDA ${ }^{6}$, KEI SATOH ${ }^{6}$, \\ YUKITOSHI AOYAGI ${ }^{7}$, YOSHINORI NOZAWA ${ }^{8}$ and YOSHIJI YAMADA ${ }^{9}$
}

\begin{abstract}
${ }^{1}$ Department of Cardiovascular Medicine, Gifu Prefectural Tajimi Hospital, Tajimi; ${ }^{2}$ Department of Cardiovascular Medicine, Inabe General Hospital, Inabe; ${ }^{3}$ Department of Cardiology, Japanese Red Cross Nagoya First Hospital, Nagoya; ${ }^{4}$ Department of Cardiology, Gifu Prefectural General Medical Center, Gifu; ${ }^{5}$ Department of Internal Medicine, Hirosaki Stroke Center, Hirosaki; ${ }^{6}$ Department of Vascular Biology, Institute of Brain Science, Hirosaki University Graduate School of Medicine, Hirosaki; ${ }^{7}$ Department of Genomics for Longevity and Health, Tokyo Metropolitan Institute of Gerontology, Tokyo; ${ }^{8}$ Gifu International Institute of Biotechnology and Tokai Gakuin University, Kakamigahara; ${ }^{9}$ Department of Human Functional Genomics, Life Science Research Center, Mie University, Tsu, Japan
\end{abstract}

Received October 29, 2009; Accepted December 28, 2009

DOI: 10.3892/ijmm_00000400

\begin{abstract}
Although chronic kidney disease (CKD) is recognized as an important risk factor for myocardial infarction (MI), genetic factors underlying predisposition to MI in individuals with or without CKD remain largely unknown. The aim of the present study was to identify genetic variants that confer susceptibility to $\mathrm{MI}$ in individuals with or without CKD in order to allow prediction of genetic risk for such individuals separately. The study population comprised a total of 4344 individuals, including 1247 individuals with CKD (506 subjects with MI and 741 controls) and 3097 individuals without CKD (833 subjects with MI and 2264 controls). The 150 polymorphisms examined in this study were selected by genome-wide association studies of ischemic stroke and MI with the use of the GeneChip Human Mapping 500K Array Set (Affymetrix) and determined by a method that combines the polymerase chain reaction and sequencespecific oligonucleotide probes with suspension array technology. In individuals with CKD, no polymorphism was significantly related to MI. In individuals without CKD, an initial screen by the Chi-square test revealed that the $\mathrm{C} \rightarrow \mathrm{T}$ polymorphism of CLEC16A (rs9925481) and the $\mathrm{A} \rightarrow \mathrm{G}$ polymorphism of LAMA3 (rs12373237) were significantly (false discovery rate for allele frequencies of $<0.05$ ) associated
\end{abstract}

Correspondence to: Dr Yoshiji Yamada, Department of Human Functional Genomics, Life Science Research Center, Mie University, 1577 Kurima-machiya, Tsu, Mie 514-8507, Japan E-mail: yamada@gene.mie-u.ac.jp

Key words: myocardial infarction, coronary heart disease, atherosclerosis, chronic kidney disease, genetics, polymorphism with MI. Subsequent multivariable logistic regression analysis with adjustment for covariates revealed that the $\mathrm{C} \rightarrow \mathrm{T}$ polymorphism of CLEC16A (dominant model; $\mathrm{P}=0.0003$; odds ratio, 0.66) and the $\mathrm{A} \rightarrow \mathrm{G}$ polymorphism of LAMA3 (recessive model; $\mathrm{P}=0.0087$; odds ratio, 0.75 ) were significantly $(\mathrm{P}<0.05)$ associated with MI. A stepwise forward selection procedure also revealed that these polymorphisms were significant and independent determinants of MI. CLEC16A and $L A M A 3$ may be susceptibility loci for MI in Japanese individuals without CKD. Determination of genotypes for CLEC16A and LAMA3 may prove informative for assessment of the genetic risk for $\mathrm{MI}$ in such individuals.

\section{Introduction}

Myocardial infarction (MI) is a multifactorial and polygenic disease, and is strongly influenced by a genetic component (1). Although recent genome-wide association studies (GWAS) have implicated various candidate genes underlying predisposition to MI (2-7), the genes that confer susceptibility to this condition remain to be identified definitively.

It is increasingly recognized that chronic kidney disease (CKD) is an independent risk factor not only for end-stage renal disease but also for atherosclerotic cardiovascular diseases including MI $(8,9)$. Individuals with CKD have a 1.9 to 2.9 times higher risk for the development of MI compared to the general population without CKD (10). Although epidemiological studies have suggested that genetic factors and geneenvironment interactions may contribute to the development of MI $(11,12)$, genetic variants underlying predisposition to MI in individuals with or without CKD remain largely unknown.

We previously showed that a polymorphism of $F 7$ was significantly associated with MI among individuals with CKD (13). To further examine whether the association of polymorphisms with MI is influenced by CKD, we 
performed an association study for 150 polymorphisms of 144 candidate genes and MI in a total of 4344 Japanese individuals, including 1247 subjects with CKD and 3097 subjects without this condition. The purpose of the present study was to identify genetic variants that confer susceptibility to MI in Japanese individuals with or without CKD in order to allow prediction of genetic risk for such individuals separately.

\section{Materials and methods}

Study population. The study population comprised a total of 4344 unrelated Japanese individuals who either visited outpatient clinics of or were admitted to one of the participating hospitals (Gifu Prefectural General Medical Center and Gifu Prefectural Tajimi Hospital in Gifu Prefecture, Japan; Hirosaki University Hospital, Reimeikyo Rehabilitation Hospital, and Hirosaki Stroke Center in Aomori Prefecture, Japan) between October 2002 and March 2008 with various symptoms or for an annual health check-up, or who were recruited to a population-based prospective cohort study of aging and agerelated diseases in Nakanojo, Gunma Prefecture, Japan. Estimated glomerular filtration rate (eGFR) was calculated with the use of the simplified prediction equation proposed by the Japanese Society of Nephrology and based on that described in the Modification of Diet in Renal Disease Study (14): eGFR $\left(\mathrm{ml} \mathrm{min}-11.73 \mathrm{~m}^{-2}\right)=194 \mathrm{x}$ [age (years) $]^{-0.287} \mathrm{x}$ [serum creatinine $(\mathrm{mg} / \mathrm{dl})]^{-1.094}$ (x 0.739 if female). The National Kidney Foundation - Kidney Disease Outcome Quality Initiative guidelines recommend a dianosis of CKD if eGFR is $<60 \mathrm{ml} \mathrm{min}{ }^{-1} 1.73 \mathrm{~m}^{-2}(15)$, on the basis of which 1247 individuals were diagnosed with CKD and 3097 individuals whose eGFR of $\geq 60 \mathrm{ml} \mathrm{min}^{-1} 1.73 \mathrm{~m}^{-2}$ were diagnosed without CKD in the present study.

The 1247 individuals with CKD comprised 506 subjects with MI and 741 controls. The 3097 individuals without CKD comprised 833 subjects with MI and 2264 controls. The 1339 subjects with MI (1048 men, 291 women) all underwent coronary angiography and left ventriculography. The diagnosis of MI was based on typical electrocardiographic changes and on increases both in the serum activity of creatine kinase (MB isozyme) and in the serum concentration of cardiac troponin $\mathrm{T}$. The diagnosis of MI was confirmed by the presence of wall motion abnormality on left ventriculography and by identification of the responsible stenosis in any of the major coronary arteries or in the left main trunk by coronary angiography. The control subjects comprised 3005 individuals (1354 men, 1651 women) who had no history of coronary heart disease, aortic aneurysm, or peripheral arterial occlusive disease; of ischemic or hemorrhagic stroke or other cerebral diseases; or of other atherosclerotic, thrombotic, embolic or hemorrhagic disorders.

The study protocol complied with the Declaration of Helsinki and was approved by the committees on the Ethics of Human Research of Mie University Graduate School of Medicine, Hirosaki University Graduate School of Medicine, Gifu International Institute of Biotechnology, Tokyo Metropolitan Institute of Gerontology, and participating hospitals. Written informed consent was obtained from each participant.

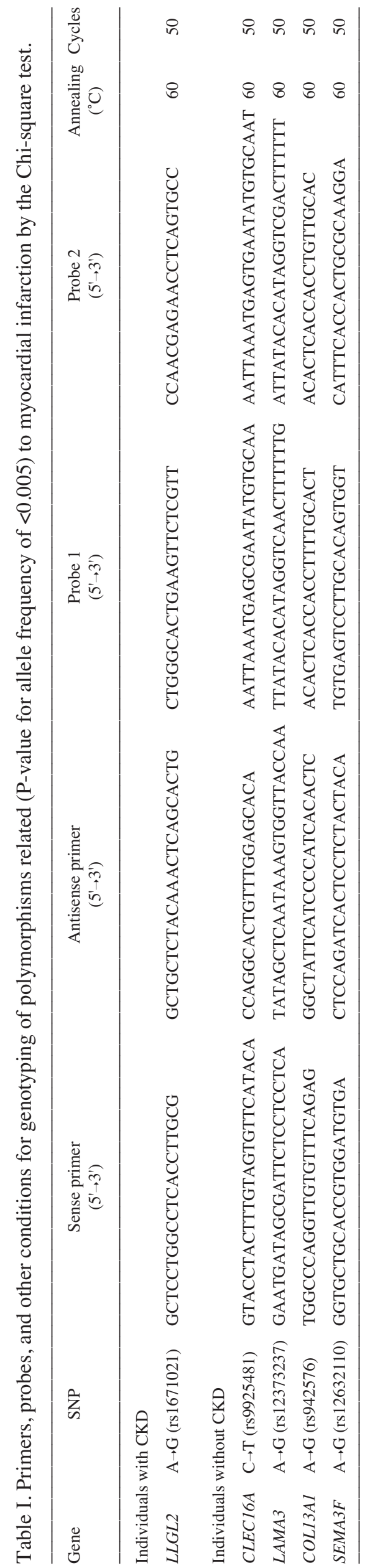


Table II. Baseline characteristics of the subjects with myocardial infarction (MI) and controls in the presence or absence of chronic kidney disease (CKD).

\begin{tabular}{|c|c|c|c|c|c|c|}
\hline \multirow[b]{2}{*}{ Characteristic } & \multicolumn{3}{|c|}{ Individuals with CKD } & \multicolumn{3}{|c|}{ Individuals without CKD } \\
\hline & $\begin{array}{c}\text { MI } \\
(\mathrm{n}=506)\end{array}$ & $\begin{array}{l}\text { Controls } \\
(\mathrm{n}=741)\end{array}$ & P-value & $\begin{array}{c}\text { MI } \\
(n=833)\end{array}$ & $\begin{array}{l}\text { Controls } \\
(\mathrm{n}=2264)\end{array}$ & P-value \\
\hline Age (years) & $70.5 \pm 8.9$ & $70.5 \pm 9.4$ & 0.9917 & $63.8 \pm 9.9$ & $65.0 \pm 10.8$ & 0.0045 \\
\hline Gender (male/female) & $395 / 111$ & $357 / 384$ & $<0.0001$ & $653 / 180$ & $997 / 1267$ & $<0.0001$ \\
\hline Body mass index $\left(\mathrm{kg} / \mathrm{m}^{2}\right)$ & $23.5 \pm 3.2$ & $23.6 \pm 3.5$ & 0.6191 & $23.9 \pm 3.3$ & $23.3 \pm 3.3$ & $<0.0001$ \\
\hline Current or former smoker $(\%)$ & 18.6 & 20.9 & 0.3100 & 29.2 & 22.4 & $<0.0001$ \\
\hline Serum creatinine $(\mu \mathrm{mol} / \mathrm{l})$ & $130.5 \pm 144.1$ & $94.4 \pm 70.3$ & $<0.0001$ & $66.3 \pm 12.3$ & $60.3 \pm 12.0$ & $<0.0001$ \\
\hline Estimated glomerular filtration rate $\left(\mathrm{ml} \mathrm{min}{ }^{-1} 1.73 \mathrm{~m}^{-2}\right)$ & $45.9 \pm 12.5$ & $50.8 \pm 8.9$ & $<0.0001$ & $78.7 \pm 15.9$ & $78.5 \pm 15.9$ & 0.6677 \\
\hline Hypertension $(\%)$ & 78.9 & 49.0 & $<0.0001$ & 66.5 & 39.3 & $<0.0001$ \\
\hline Systolic blood pressure (mmHg) & $146.7 \pm 27.9$ & $138.5 \pm 22.9$ & $<0.0001$ & $139.7 \pm 24.9$ & $136.7 \pm 20.0$ & 0.0014 \\
\hline Diastolic blood pressure $(\mathrm{mmHg})$ & $74.5 \pm 14.6$ & $78.3 \pm 13.5$ & $<0.0001$ & $74.5 \pm 14.5$ & $78.8 \pm 11.6$ & $<0.0001$ \\
\hline Diabetes mellitus (\%) & 50.8 & 19.7 & $<0.0001$ & 46.6 & 13.7 & $<0.0001$ \\
\hline Fasting plasma glucose level (mmol/l) & $7.59 \pm 3.46$ & $6.62 \pm 2.98$ & $<0.0001$ & $7.72 \pm 3.51$ & $6.43 \pm 2.71$ & $<0.0001$ \\
\hline Blood glycosylated hemoglobin (\%) & $6.53 \pm 1.77$ & $5.60 \pm 1.27$ & $<0.0001$ & $6.68 \pm 1.87$ & $5.59 \pm 1.35$ & $<0.0001$ \\
\hline Hypercholesterolemia (\%) & 29.8 & 29.6 & 0.9132 & 29.1 & 27.0 & 0.2498 \\
\hline Serum total cholesterol (mmol/l) & $5.16 \pm 0.98$ & $5.15 \pm 0.98$ & 0.9347 & $5.18 \pm 1.09$ & $5.13 \pm 0.89$ & 0.2665 \\
\hline High density lipoprotein-cholesterol (mmol/l) & $1.21 \pm 0.39$ & $1.45 \pm 0.40$ & $<0.0001$ & $1.20 \pm 0.33$ & $1.50 \pm 0.39$ & $<0.0001$ \\
\hline Serum triglycerides $(\mathrm{mmol} / \mathrm{l})$ & $1.70 \pm 0.90$ & $1.65 \pm 1.02$ & 0.3175 & $1.78 \pm 1.29$ & $1.51 \pm 0.97$ & $<0.0001$ \\
\hline
\end{tabular}

Quantitative data are means \pm SD. Hypertension: systolic blood pressure of $\geq 140 \mathrm{mmHg}$, diastolic blood pressure of $\geq 90 \mathrm{mmHg}$, or taking of antihypertensive medication. Diabetes mellitus: fasting plasma glucose level of $\geq 6.93 \mathrm{mmol} / 1$ ( $126 \mathrm{mg} / \mathrm{dl})$, blood glycosylated hemoglobin (hemoglobin $\mathrm{A}_{1 \mathrm{c}}$ ) content of $\geq 6.5 \%$, or taking of antidiabetes medication. Hypercholesterolemia: serum total cholesterol concentration of $\geq 5.72 \mathrm{mmol} / \mathrm{l}(220 \mathrm{mg} / \mathrm{dl})$ or taking of lipid-lowering medication.

Selection of polymorphisms. Our aim was to identify genetic variants associated with MI in the Japanese population in a case-control association study. A total of 150 polymorphisms examined in the present study (data not shown) were selected by GWAS of ischemic stroke (16) and MI (P-value for allele frequency $<1.0 \times 10^{-7}$ ) with the use of the GeneChip Human Mapping 500K Array Set (Affymetrix, Santa Clara, CA). We have not examined the relation of these polymorphisms to MI among individuals with or without CKD in our previous studies (17-23).

Genotyping of polymorphisms. Venous blood $(7 \mathrm{ml})$ was collected into tubes containing ethylenediaminetetraacetic acid at a final concentration of $50 \mathrm{mM}$, and genomic DNA was isolated with a kit (Genomix; Talent, Trieste, Italy). Genotypes of 150 polymorphisms were determined at G\&G Science (Fukushima, Japan) by a method that combines the polymerase chain reaction and sequence-specific oligonucleotide probes with suspension array technology (Luminex, Austin, TX, USA). Primers, probes, and other conditions for genotyping of polymorphisms related ( $\mathrm{P}$-value for allele frequency $<0.005$ ) to MI as determined by the initial Chisquare test are shown in Table I. The detailed genotyping method was described previously (24).

Statistical analysis. Quantitative data were compared between subjects with MI and controls by the unpaired Student's t-test. Categorical data were compared by the Chi-square test. Allele frequencies were estimated by the gene counting method, and the Chi-square test was used to identify departures from Hardy-Weinberg equilibrium. In the initial screen, the genotype distribution $(3 \times 2)$ and the allele frequencies $(2 \times 2)$ of each polymorphim were compared between subjects with MI and controls by the Chi-square test. Given the multiple comparisons of genotypes with MI, the false discovery rate (FDR) was calculated from the distributions of P-values of the 150 polymorphisms (25). Polymorphisms with a P-value for allele frequency of $<0.005$ were further examined by multivariable logistic regression analysis with adjustment for covariates. Such analysis was thus performed with MI as a dependent variable and independent variables including age, gender (0, woman; 1 , man), body mass index (BMI), smoking status $(0$, nonsmoker; 1 , smoker $)$, the serum concentration of creatinine, the history of hypertension, diabetes mellitus, and hypercholesterolemia ( 0 , no history; 1 , positive history), and genotype of each polymorphism; and the P-value, odds ratio, and $95 \%$ confidence interval were calculated. Each genotype was assessed according to dominant, recessive, and additive genetic models. Additive models included the additive 1 (heterozygotes versus wild-type homozygotes) and the additive 2 (variant homozygotes versus wild-type homozygote) models, which were analyzed simultaneously with a single statistical model. We also performed a stepwise forward selection procedure to examine the effects of genotypes as well as of other covariates on MI. In this analysis, each genotype was examined according to a dominant or 
Table III. Polymorphisms related (P-value for allele frequency $<0.005)$ to myocardial infarction $(\mathrm{MI})$ in individuals with or without chronic kidney disease (CKD) as determined by the Chi-square test.

\begin{tabular}{|c|c|c|c|c|c|c|c|}
\hline Gene & Polymorphisms & $\mathrm{dbSNP}$ & $\mathrm{MI}^{\mathrm{a}}$ & Controls $^{\mathrm{a}}$ & $\begin{array}{c}\text { P-value } \\
\text { (genotype) }\end{array}$ & $\begin{array}{l}\text { P-value } \\
\text { (allele) }\end{array}$ & FDR \\
\hline \multicolumn{8}{|c|}{ Individuals with CKD } \\
\hline \multirow[t]{4}{*}{$L L G L 2$} & $\mathrm{~A} \rightarrow \mathrm{G}$ & rs1671021 & & & 0.0135 & 0.0044 & 0.2532 \\
\hline & $A A$ & & $393(77.7)$ & $526(71.2)$ & & & \\
\hline & $A G$ & & $108(21.3)$ & $194(26.2)$ & & & \\
\hline & $G G$ & & $5(1.0)$ & $19(2.6)$ & & & \\
\hline \multicolumn{8}{|c|}{ Individuals without CKD } \\
\hline \multirow[t]{4}{*}{ CLEC16A } & $\mathrm{C} \rightarrow \mathrm{T}$ & rs9925481 & & & 0.0005 & 0.0001 & 0.0148 \\
\hline & $C C$ & & $674(81.2)$ & $1659(74.5)$ & & & \\
\hline & $C T$ & & $145(17.5)$ & $524(23.6)$ & & & \\
\hline & $T T$ & & 11 (1.3) & $43(1.9)$ & & & \\
\hline \multirow[t]{4}{*}{ LAMA3 } & $\mathrm{A} \rightarrow \mathrm{G}$ & rs 12373237 & & & 0.0003 & 0.0001 & 0.0148 \\
\hline & $A A$ & & 14 (1.7) & 33 (1.5) & & & \\
\hline & $A G$ & & $204(24.6)$ & $404(18.1)$ & & & \\
\hline & $G G$ & & $612(73.7)$ & $1788(80.4)$ & & & \\
\hline \multirow[t]{4}{*}{ COL13A1 } & $\mathrm{A} \rightarrow \mathrm{C}$ & rs 942576 & & & 0.0077 & 0.0015 & 0.0735 \\
\hline & $A A$ & & $25 \quad(3.0)$ & $47 \quad(2.1)$ & & & \\
\hline & $A C$ & & $215(26.0)$ & $479(21.5)$ & & & \\
\hline & $C C$ & & $590(71.0)$ & $1707(76.4)$ & & & \\
\hline \multirow[t]{4}{*}{ SEMA3F } & $\mathrm{A} \rightarrow \mathrm{G}$ & rs 12632110 & & & 0.0017 & 0.0019 & 0.0735 \\
\hline & $A A$ & & $216(26.0)$ & $449(20.1)$ & & & \\
\hline & $A G$ & & $387(46.7)$ & $1108(49.7)$ & & & \\
\hline & $G G$ & & $226(27.3)$ & $674(30.2)$ & & & \\
\hline
\end{tabular}

${ }^{a}$ Numbers in parentheses are percentages.

recessive model on the basis of statistical significance in the multivariable logistic regression analysis. The P-levels for inclusion in and exclusion from the model were 0.25 and 0.1 , respectively. With the exception of the initial screen by the Chi-square test (FDR $<0.05$ ), a P-value of $<0.05$ was considered statistically significant. Statistical significance was examined by two-sided tests performed with JMP version 6.0 and JMP Genomics version 3.2 softwares (SAS Institute, Cary, NC).

\section{Results}

Baseline characteristics of the subjects with MI and controls in the presence or absence of CKD are shown in Table II. Among individuals with CKD, the frequency of male subjects, serum concentration of creatinine, systolic blood pressure, fasting plasma glucose level, blood glycosylated hemoglobin content, and the prevalence of hypertension and diabetes mellitus were greater, whereas eGFR, diastolic blood pressure, and serum concentration of high density lipoprotein (HDL)cholesterol were lower in subjects with MI than in controls. Among individuals without CKD, the frequency of male subjects, BMI, the percentage of smokers, serum concentrations of creatinine and triglycerides, systolic blood pressure, fasting plasma glucose level, blood glycosylated hemoglobin
Table IV. Hardy-Weinberg P-values in subjects with myocardial infarction (MI) and controls.

\begin{tabular}{|c|c|c|c|c|}
\hline Gene & SNP & $\mathrm{dbSNP}$ & MI & Controls \\
\hline \multicolumn{5}{|c|}{ Individuals with CKD } \\
\hline$L L G L 2$ & $\mathrm{~A} \rightarrow \mathrm{G}$ & rs 1671021 & 0.4173 & 0.8258 \\
\hline \multicolumn{5}{|c|}{ Individuals without CKD } \\
\hline CLEC16A & $\mathrm{C} \rightarrow \mathrm{T}$ & rs9925481 & 0.3186 & 0.8283 \\
\hline$L A M A 3$ & $A \rightarrow G$ & rs12373237 & 0.5231 & 0.0664 \\
\hline COL13A1 & $\mathrm{A} \rightarrow \mathrm{C}$ & rs942576 & 0.3195 & 0.0526 \\
\hline SEMA3F & $A \rightarrow G$ & rs 12632110 & 0.0566 & 0.8693 \\
\hline
\end{tabular}

content, and the prevalence of hypertension and diabetes mellitus were greater, whereas age, diastolic blood pressure, and serum concentration of HDL-cholesterol were lower in subjects with MI than in controls.

The genotype distributions and allele frequencies of polymorphisms were compared between subjects with MI and controls by the Chi-square test (Table III). For individuals with CKD, although one polymorphism was related (P-value for allele frequency of $<0.005$ ) to the prevalence of 
Table VI. Effects of genotypes and other characteristics on the prevalence of myocardial infarction as determined by a stepwise forward selection procedure.

\begin{tabular}{lrc}
\hline Characteristics & P-value & $\mathrm{R}^{2}$ \\
\hline Individuals with CKD & & \\
Diabetes mellitus & $<0.0001$ & 0.0784 \\
Male gender & $<0.0001$ & 0.0607 \\
Hypertension & $<0.0001$ & 0.0521 \\
Smoking & $<0.0001$ & 0.0100 \\
LLGL2 (dominant model, $A G+G G$ versus $A A)$ & 0.0130 & 0.0037 \\
Serum creatinine concentration & 0.0133 & 0.0036 \\
& & \\
Individuals without CKD & $<0.0001$ & 0.0981 \\
Diabetes mellitus & $<0.0001$ & 0.0634 \\
Male gender & $<0.0001$ & 0.0243 \\
Hypertension & 0.0003 & 0.0038 \\
Age & 0.0003 & 0.0036 \\
CLEC16A (dominant model, $C T+T T$ versus $C C$ ) & 0.0022 & 0.0027 \\
SEMA3F (dominant model, $A G+G G$ versus $A A)$ & 0.0094 & 0.0019 \\
LAMA3 (recessive model, $G G$ versus $A A+A G$ ) & 0.0163 & 0.0016 \\
Smoking & 0.0221 & 0.0015 \\
COL13A1 (recessive model, $C C$ versus $A A+A C$ ) & & \\
\hline
\end{tabular}

$\mathrm{R}^{2}$, contribution rate.

MI, it was not significantly associated with this condition. For individuals without CKD, four polymorphisms were related to the prevalence of MI. Among these polymorphisms, the $\mathrm{C} \rightarrow \mathrm{T}$ polymorphism of CLEC16A (rs9925481) and the $\mathrm{A} \rightarrow \mathrm{G}$ polymorphism of LAMA3 (rs12373237) were significantly (FDR for allele frequency of $<0.05$ ) associated with MI. The genotype distributions for the five polymorphisms related to MI are also shown in Table III. Genotype distributions of these polymorphisms related to MI were in Hardy-Weinberg equilibrium for the subjects with MI and controls (Table IV).

Multivariable logistic regression analysis with adjustment for age, gender, BMI, smoking status, serum concentration of creatinine, and the prevalence of hypertension, diabetes mellitus, and hypercholesterolemia revealed that the $A \rightarrow G$ polymorphism of LLGL2 (all genetic models) was significantly $(\mathrm{P}<0.05)$ associated with $\mathrm{MI}$ among individuals with $\mathrm{CKD}$ and that the $\mathrm{C} \rightarrow \mathrm{T}$ polymorphism of CLEC16A (dominant and additive 1 models), the $\mathrm{A} \rightarrow \mathrm{G}$ polymorphism of $L A M A 3$ (recessive model), the $\mathrm{A} \rightarrow \mathrm{C}$ polymorphism of $\mathrm{COL13A1}$ (recessive model), and the $\mathrm{A} \rightarrow \mathrm{G}$ polymorphism of $S E M A 3 F$ (dominant and additive 1 and 2 models) were significantly associated with MI among individuals without CKD (Table V). The $G$ allele of $L L G L 2$, the $T$ allele of CLEC16A, the $G$ allele of $L A M A 3$, the $C$ allele of $C O L 13 A 1$, and the $G$ allele of $S E M A 3 F$ were all protective against MI.

A stepwise forward selection procedure was performed to examine the effects of genotypes for the polymorphisms related to MI by the Chi-square test as well as of age, gender, BMI, smoking status, serum concentration of creatinine, and 
the prevalence of hypertension, diabetes mellitus, and hypercholesterolemia on MI (Table VI). For individuals with $\mathrm{CKD}$, diabetes mellitus, male gender, hypertension, smoking, LLGL2 (dominant model), serum creatinine concentration, in descending order of statistical significance, were significant $(\mathrm{P}<0.05)$ and independent determinants of MI. For individuals without $\mathrm{CKD}$, diabetes mellitus, male gender, hypertension, age, CLEC16A (dominant model), SEMA3F (dominant model), LAMA3 (recessive model), smoking, and COL13A1 (recessive model), in descending order of statistical significance, were significant and independent determinants of MI (Table VI).

\section{Discussion}

We examined the possible relations of 150 polymorphisms to the prevalence of MI in a total of 4344 Japanese individuals with or without CKD. Our association study with three steps of analysis (Chi-square test, multivariable logistic regression analysis with adjustment for covariates, and a stepwise forward selection procedure) revealed that the $\mathrm{C} \rightarrow \mathrm{T}$ polymorphism of CLEC16A (rs9925481) and the $\mathrm{A} \rightarrow \mathrm{G}$ polymorphism of LAMA3 (rs12373237) were significantly associated with MI in Japanese individuals without CKD.

C-type lectin domain family 16, member A (CLEC16A) has been detected mainly in immune cells including $B$ lymphocytes and natural killer cells (26). Two independent GWAS in Northern European (27) and American (28) populations showed that several variants of CLEC16A were significantly associated with type 1 diabetes mellitus. It was hypothesized that the altered protein structure could elicit an autoimmune response, resulting in the destruction of islet cells of the pancreas as noted in type 1 diabetes mellitus. We now showed that the $\mathrm{C} \rightarrow \mathrm{T}$ polymorphism in intron 11 of CLEC16A (rs9925481) was significantly associated with the prevalence of MI in Japanese individuals without CKD, with the $T$ allele protecting against this condition. The effect of CLEC16A on glucose metabolism might account for its association with $\mathrm{MI}$, although the underlying mechanism remains unclear.

Laminins are members of the glycoprotein family that are the main components of the multifunctional extracellular matrix proteins regulating adhesion, motility, gene expression, and apoptosis (29). Targeted disruption of the laminin, $\alpha 3$ gene (LAMA3) in mice caused the formation of a lethal epidermal blistering condition similar to human junctional epidermolysis bullosa (29). LAMA3 was also shown to play an important role in the regulation of endothelial wound healing (30). We now showed that the $\mathrm{A} \rightarrow \mathrm{G}$ polymorphism in intron 30 of LAMA3 (rs12373237) was significantly associated with the prevalence of MI in Japanese individuals without CKD, with the $G$ allele protecting against this condition. The altered endothelial function by means of adhesion and cell migration might be implicated in the pathogenesis of MI, although the functional relevance of this polymorphism with the pathogenesis of MI remains to be elucidated.

Our results suggest that $L L G L 2$ in individuals with CKD, and COL13A1 and SEMA3F in individuals without CKD are also candidate genes for MI in Japanese individuals. Lethal giant larvae homolog 2 (LLGL2) is involved in epithelial cell polarity, asymmetric cell division, and hemidesmosome formation through the association with submembranous actin cytoskeleton of the basolateral cell domain $(31,32)$. Collagen, type XIII, $\alpha 1$ (COL13A1) is a constituent of type XIII collagen. It is a transmembrane protein which belongs to a non-fibrillar collagen group (33), and is expressed in various tissues including the bone, muscle, and heart, being implicated in cellular adhesion and migration $(34,35)$. Sema domain, immunoglobulin domain, short basic domain, secreted, $3 \mathrm{~F}$ (SEMA3F) is a member of the class 3 semaphorins and plays an important role in endothelial cell functions in vasculature (36). Inhibition of SEMA3F expression by small interfering RNA affected the intracellular localization and function of gap junction protein, $\alpha 1,43 \mathrm{kDa}$ which is expressed in medial smooth muscle cells of the normal vascular wall $(37,38)$, being increased at early stages of atherosclerosis and reduced in advanced lesion (39).

There are several limitations to the present study. (i) It is possible that one or more of the polymorphisms associated with MI in the present study are in linkage disequilibrium with other polymorphisms in the same gene or in other nearby genes that are actually responsible for the development of this condition. (ii) The functional relevance of identified polymorphisms to gene transcription or to protein structure or function was not determined in the present study. (iii) Although we adopted the criterion of FDR $<0.05$ for association to compensate for the multiple comparisons of genotypes with MI, it is not possible to exclude completely potential statistical errors such as false-positives. (iv) Given that the results of the present study were not replicated, validation of our findings will require their replication with other independent subject panels.

In conclusion, our present results suggest that CLEC16A and $L A M A 3$ may be susceptibility loci for MI in Japanese individuals without CKD. Determination of genotypes for CLEC16A and LAMA3 may prove informative for assessment of the genetic risk for MI in such individuals. Given that genetic variants that confer susceptibility to MI differed between individuals with CKD and those without this condition, stratification of subjects according to CKD may thus be important for personalized prevention of MI based on genetic information.

\section{Acknowledgements}

This work was supported by Grants-in-Aid for Scientific Research from the Ministry of Education, Culture, Sports, Science, and Technology of Japan (nos. 18209023, 18018021, and 19659149 to Y.Y.).

\section{References}

1. Nora JJ, Lortscher RH, Spangler RD, et al: Geneticepidemiologic study of early-onset ischemic heart disease. Circulation 61: 503-508, 1980.

2. Wellcome Trust Case Control Consortium: Genome-wide association study of 14,000 cases of seven common diseases and 3,000 shared controls. Nature 447: 661-678, 2007.

3. Myocardial Infarction Genetics Consortium: Genome-wide association of early-onset myocardial infarction with single nucleotide polymorphisms and copy number variants. Nat Genet 41: 334-341, 2009 
4. Erdmann J, Grosshennig A, Braund PS, et al: New susceptibility locus for coronary artery disease on chromosome $3 \mathrm{q} 22.3$. Nat Genet 41: 280-282, 2009

5. Helgadottir A, Thorleifsson G, Manolescu A, et al: A common variant on chromosome 9p21 affects the risk of myocardial infarction. Science 316: 1491-1493, 2007.

6. McPherson R, Pertsemlidis A, Kavaslar N, et al: A common allele on chromosome 9 associated with coronary heart disease. Science 316: 1488-1491, 2007.

7. Samani NJ, Erdmann J, Hall AS, et al: Genomewide association analysis of coronary artery disease. N Engl J Med 357: 443-453 2007.

8. Best PJ, Lennon R, Ting HH, et al: The impact of renal insufficiency on clinical outcomes in patients undergoing percutaneous coronary interventions. J Am Coll Cardiol 39: 1113-1119, 2002.

9. Mann JF, Gerstein HC, Pogue J, et al: Renal insufficiency as a predictor of cardiovascular outcomes and the impact of ramipril: the HOPE randomized trial. Ann Intern Med 134: 629-636, 2001 .

10. Anavekar NS, McMurray JJ, Velazquez EJ, et al: Relation between renal dysfunction and cardiovascular outcomes after myocardial infarction. N Engl J Med 351: 1285-1295, 2004.

11. Arnett DK, Baird AE, Barkley RA, et al: Relevance of genetics and genomics for prevention and treatment of cardiovascular disease: a scientific statement from the American Heart Association Council on Epidemiology and Prevention, the Stroke Council, and the Functional Genomics and Translational Biology Interdisciplinary Working Group. Circulation 115: 2878-2901, 2007.

12. Kullo IJ and Ding K: Mechanisms of disease: The genetic basis of coronary heart disease. Nat Clin Pract Cardiovasc Med 4: 558-569, 2007

13. Fujimaki T, Kato K, Yoshida $\mathrm{T}$, et al: Association of genetic variants with myocardial infarction in Japanese individuals with chronic kidney disease. Thromb Haemost 101: 963-968, 2009.

14. Matsuo S, Imai E, Horio M, et al: Revised equations for estimated GFR from serum creatinine in Japan. Am J Kidney Dis 53: 982-992, 2009.

15. Levey AS, Eckardt KU, Tsukamoto Y, et al: Definition and classification of chronic kidney disease: a position statement from Kidney Disease: Improving Global Outcomes (KDIGO). Kidney Int 67: 2089-2100, 2005.

16. Yamada Y, Fuku N, Tanaka M, et al: Identification of CELSR1 as a susceptibility gene for ischemic stroke in Japanese individuals by a genome-wide association study. Atherosclerosis 207: 144-149, 2009

17. Yamada Y, Izawa H, Ichihara S, et al: Prediction of the risk of myocardial infarction from polymorphisms in candidate genes. N Engl J Med 347: 1916-1923, 2002.

18. Yamada Y, Kato K, Oguri M, et al: Genetic risk for myocardial infarction determined by polymorphisms of candidate genes in a Japanese population. J Med Genet 45: 216-221, 2008.

19. Yamada Y, Matsuo H, Segawa T, et al: Assessment of genetic risk for myocardial infarction. Thromb Haemost 96: 220-227, 2006.

20. Yoshida T, Kato K, Yokoi K, et al: Association of genetic variants with myocardial infarction in Japanese individuals with different lipid profiles. Int J Mol Med 25: 607-616, 2010.

21. Yoshida T, Kato K, Yokoi K, et al: Association of genetic variants with myocardial infarction in individuals with or without hypertension or diabetes mellitus. Int J Mol Med 24: 701-709, 2009
22. Yoshida T, Yajima K, Hibino T, et al: Association of gene polymorphisms with myocardial infarction in individuals with different lipid profiles. Int J Mol Med 20: 581-590, 2007.

23. Nishihama K, Yamada Y, Matsuo H, et al: Association of gene polymorphisms with myocardial infarction in individuals with or without conventional coronary risk factors. Int J Mol Med 19: 129-141, 2007.

24. Itoh Y, Mizuki N, Shimada T, et al: High-throughput DNA typing of HLA-A, -B, $-\mathrm{C}$, and -DRB1 loci by a PCR-SSOPLuminex method in the Japanese population. Immunogenetics 57: 717-729, 2005.

25. Benjamini Y and Hochberg Y: Controlling the false discovery rate: a practical and powerful approch to multiple testing. J Royal Stat Soc Ser B 57: 289-300, 1995.

26. Skinningsrud B, Husebye ES, Pearce SH, et al: Polymorphisms in CLEC16A and CIITA at $16 \mathrm{p} 13$ are associated with primary adrenal insufficiency. J Clin Endocrinol Metab 93: 3310-3317, 2008.

27. Todd JA, Walker NM, Cooper JD, et al: Robust associations of four new chromosome regions from genome-wide analyses of type 1 diabetes. Nat Genet 39: 857-864, 2007.

28. Hakonarson H, Grant SF, Bradfield JP, et al: A genome-wide association study identifies KIAA0350 as a type 1 diabetes gene. Nature 448: 591-594, 2007.

29. Ryan MC, Lee K, Miyashita Y, et al: Targeted disruption of the LAMA3 gene in mice reveals abnormalities in survival and late stage differentiation of epithelial cells. J Cell Biol 145: 1309-1323, 1999.

30. Tzu J and Marinkovich MP: Bridging structure with function: structural, regulatory, and developmental role of laminins. Int $\mathrm{J}$ Biochem Cell Biol 40: 199-214, 2008.

31. Lisovsky M, Dresser $\mathrm{K}$, Baker $\mathrm{S}$, et al: Cell polarity protein Lg12 is lost or aberrantly localized in gastric dysplasia and adenocarcinoma: an immunohistochemical study. Mod Pathol 22: 977-984, 2009.

32. Bilder D: Epithelial polarity and proliferation control: links from the Drosophila neoplastic tumor suppressors. Genes Dev 18: 1909-1925, 2004.

33. Kvist AP, Latvanlehto A, Sund M, et al: Complete exon-intron organization and chromosomal location of the gene for mouse type XIII collagen (col13a1) and comparison with its human homologue. Matrix Biol 18: 261-274, 1999.

34. Kvist AP, Latvanlehto A, Sund M, et al: Lack of cytosolic and transmembrane domains of type XIII collagen results in progressive myopathy. Am J Pathol 159: 1581-1592, 2001.

35. Ylonen R, Kyronlahti T, Sund M, et al: Type XIII collagen strongly affects bone formation in transgenic mice. J Bone Miner Res 20: 1381-1393, 2005.

36. Bielenberg DR and Klagsbrun M: Targeting endothelial and tumor cells with semaphorins. Cancer Metastasis Rev 26: 421-431, 2007

37. Kawasaki Y, Kubomoto A and Yamasaki H: Control of intracellular localization and function of $\mathrm{Cx} 43$ by SEMA3F. J Membr Biol 217: 53-61, 2007.

38. Severs NJ, Rothery S, Dupont E, et al: Immunocytochemical analysis of connexin expression in the healthy and diseased cardiovascular system. Microsc Res Tech 52: 301-322, 2001.

39. Blackburn JP, Peters NS, Yeh HI, et al: Upregulation of connexin43 gap junctions during early stages of human coronary atherosclerosis. Arterioscler Thromb Vasc Biol 15 $1219-1228,1995$ 